\title{
USO DE DROGAS E DESEMPENHO ESCOLAR ENTRE JOVENS E ADOLESCENTES DO ENSINO MÉDIO DE UMA ESCOLA PÚBLICA DE PIRES DO RIO - GO
}

W. P. S. D'orazio ${ }^{1}$, S. A. Carvalho ${ }^{1}$, T. H. Lima ${ }^{1}$, A. A. T. Borges ${ }^{1}$, M. C. Picoli ${ }^{1}$, A. C. L. Marques ${ }^{1}$, K. F. G. Santos ${ }^{1}$, J. F. Oliveira ${ }^{1}$, E. S. Gregório ${ }^{1}$, P. S. Resende ${ }^{2}$, M. A. S. B. Alves ${ }^{2}$, R. C. Gonçalves ${ }^{2}$, G. Malafaia ${ }^{3}$

${ }^{1}$ Curso de Licenciatura em Ciências Biológicas, bolsista PIBID/CAPES - Subprojeto de Ciências Biológicas, Instituto Federal Goiano - Câmpus Urutaí, GO, Brasil; ${ }^{2}$ Supervisores e bolsistas PIBID/CAPES - Subprojeto de Ciências Biológicas, Instituto Federal Goiano - Câmpus Urutaí, GO, Brasil; ${ }^{3}$ Professor do Departamento de Ciências

Biológicas do Instituto Federal Goiano - Câmpus Urutaí, Coordenador do PIBID/CAPES - Subprojeto de Ciências Biológicas e Pesquisador do Núcleo de Pesquisa em Ciências Ambientais e Biológicas.

E-mail: guilhermeifgoiano@gmail.com

Artigo submetido em junho/2013 e aceito em outubro/2013

\section{RESUMO}

Este estudo teve como objetivo estimar a prevalência do uso de drogas por discentes de uma escola da rede estadual de Pires do Rio, Goiás, associando-a ao desempenho escolar dos mesmos. Participaram da pesquisa 371 discentes do ensino médio, com idade entre 14 e 34 anos, dos turnos matutino e noturno que responderam a um questionário anônimo coletivamente em sala de aula. As drogas mais consumidas, alguma vez na vida, foram: álcool $(95,8 \%)$, tabaco $(46,1 \%)$, maconha
$(3,5 \%)$, solventes $(4 \%)$, cocaína $(4 \%)$ e crack $(0,5 \%)$. A partir deste estudo, foi possível perceber que os índices de reprovação apresentados, nos períodos investigados, foi elevado, quando associado aos discentes que já tiveram contato com algum tipo de droga, o que reforça a hipótese de que o uso de tais substâncias pode interferir no desempenho escolar dos alunos. Considerase necessário a implantação de políticas de intervenção contra o uso de drogas, a fim de minimizar o problema.

PALAVRAS-CHAVE: Uso de drogas, discentes, desempenho escolar.

\section{DRUG USE AND SCHOOL PERFORMANCE AMONG YOUNG TEENAGERS AND THE SCHOOL OF A PUBLIC SCHOOL OF PIRES DO RIO - GO}

\begin{abstract}
This study aimed to estimate the prevalence of drug use by students in a state school of Pires do Rio, Goiás, associating it with the performance of the same school. Participants were 371 high school students, aged 14 to 34 , the morning and evening shifts who responded to an anonymous collectively in the classroom. The most frequently used drugs at least once in life, were alcohol $(95.8 \%)$, tobacco $(46.1 \%)$, marijuana $(3.5 \%)$, solvents $(4 \%)$, cocaine $(4 \%)$ and crack $(0.5 \%)$. From this study, it
\end{abstract}

was possible that the failure rates presented in the periods investigated, was high when associated with students who have had contact with any drugs, which reinforces the hypothesis that the use of such substances can interfere the academic performance of students. It is considered necessary to implement intervention policies against drug use in order to minimize the problem

KEYWORDS: Drug Use, students, school performance. 


\section{USO DE DROGAS E DESEMPENHO ESCOLAR ENTRE JOVENS E ADOLESCENTES DO ENSINO MÉDIO DE UMA ESCOLA PÚBLICA DE PIRES DO RIO - GO.}

\section{INTRODUÇÃO}

Droga, em seu contexto farmacológico, abrange qualquer substância química, natural ou sintética, que tem a propriedade de atuar, nos indivíduos que as consomem, provocando alterações psíquicas e físicas, podendo levar a problemas de dependência (OBID, 2011).

A prática humana de consumir drogas é milenar e universal (TAVARES et al., 2001). O uso de tais substâncias, para as mais diversas finalidades (como para fins terapêuticos, de controle de pragas, de doenças, de forma divina, demonizada, dentre outras), é tão antigo quanto à própria humanidade (SANCEVERINO e ABREU, 2003). Porém, nas últimas décadas, o uso e abuso de drogas, lícitas ou ilícitas, destaca-se como um problema mundial de saúde pública em função da sua crescente prevalência e dos impactos sociais e sanitários (SANCEVERINO e ABREU, 2003, TAVARES et al., 2004; SAMPAIO et al., 2009).

Sabe-se que, uma das etapas mais importante do desenvolvimento humano, que suscita preocupações em relação ao uso de substâncias psicoativas como álcool e drogas ilícitas é a adolescência, uma vez que esta fase da vida, caracterizada por transformações biopsicossociais, constitui maior vulnerabilidade e exposição ao uso de tais substâncias (BUCHER, 1992; ALMEIDA FILHO et al., 2007; MALTA et al., 2011) em virtude dos conflitos pessoais e interpessoais típico desta faixa etária.

Estudos realizados em algumas cidades brasileiras, com enfoque no uso de drogas na adolescência, como os de Tavares et al. (2001) e Strauch et al. (2009), apontam que o consumo destas substância está associado com o desempenho escolar dos jovens. Neste sentido, a constante exposição dos adolescentes às drogas, tem se constituído, não apenas, um desafio aos profissionais da área da saúde como também da educação.

Neste contexto, o presente trabalho objetivou estimar a prevalência de uso de drogas, em uma amostra representativa de jovens e adolescentes do ensino médio de uma escola pública de Pires do Rio, Goiás, associando-o ao desempenho escolar dos mesmos. Entende-se que as informações obtidas neste estudo poderão ser úteis para o desenvolvimento de ações de prevenção do abuso e dependência dessas substâncias, bem como contribuir para discussões voltadas ao uso de drogas entre adolescentes escolares.

\section{MATERIAL E MÉTODOS}

\section{- Área de estudo e população alvo}

O presente estudo foi desenvolvido no Colégio Estadual Professor Ivan Ferreira (CEPIF), localizado na Avenida Egídio Francisco Rodrigues, no centro da cidade de Pires do Rio, Goiás. A referida instituição de ensino foi criada pela Lei estadual no 4.053, de 06 de julho de 1962. Atualmente, o CEPIF oferece à comunidade o ensino de nível médio, funcionando nos períodos diurno e noturno. No momento da pesquisa, a instituição de ensino contabilizava 17 turmas, totalizando 483 matrículas de alunos regulares. Foram entrevistados 371 alunos do ensino médio, o que corresponde $76 \%$ do número total de alunos matriculados nos turnos estudados 
(matutino e noturno).

Pode-se dizer que o CEPIF atende uma diversidade grande de alunos. São atendidos alunos com deficiência física, mental, auditiva, visual, alunos superdotados/talentosos, e alunos que aparentemente não apresentam nenhum tipo de deficiência, porém possuem dificuldades de aprendizagem. A clientela do referido colégio vem de uma classe média baixa, sendo $20 \%$ da zona rural e $70 \%$ da zona urbana de Pires do Rio e $10 \%$ de outros municípios.

Em relação à infraestrutura o CEPIF possui sanitários dentro e fora dos prédios da escola, biblioteca equipada com equipamentos audiovisuais e bibliográficos atualizados, cozinha, laboratório de informática, de Ciências, sala específica para a direção do colégio e para os docentes, além de quadra poliesportiva para o desenvolvimento de atividades físicas. Ademais, a escola oferece alimentação aos estudantes, água filtrada e mostra-se acessível aos portadores de deficiência.

\section{- $O$ instrumento de coleta de dados}

Para a coleta de dados, utilizou-se um questionário investigativo, autopreenchível e sem identificação, composto por questões objetivas e discursivas, elaborado com base no instrumento elaborado por Tavares et al. (2001). Por meio do questionário, buscou-se informações diversas quanto ao perfil sociodemográfico do público investigado (à idade, sexo, raça, estado civil, religião, renda familiar), possível uso de drogas, com ou sem fins médicos (álcool, cigarro, maconha, crack, cocaína, solvente, tranquilizante, emagrecedor e sedativo) e associação entre uso de drogas e reprovação escolar.

\section{- Aplicação do questionário investigativo}

A coleta de dados foi realizada entre os meses de março a abril de 2012, por uma equipe de 10 graduandos do curso de Licenciatura em Ciências Biológicas do Instituto Federal Goiano Câmpus Urutaí, bolsistas do Programa Institucional de Bolsas de Iniciação a Docência (PIBID). A aplicação do instrumento foi feita coletivamente, em sala de aula, com a colaboração do professor regente que se encontrava na sala. Os pesquisadores retornaram a escola, em três ocasiões subsequentes, para aplicar o questionário aos alunos que estavam ausentes durante o momento da aplicação do questionário.

\section{- Análise dos dados}

Para análise exploratória dos dados, as informações coletadas foram organizadas em planilhas eletrônicas específicas e posteriormente analisadas por meio de estatística descritiva. Deve-se ressaltar que, algumas respostas dos alunos, foram analisadas de modo separado, por sexo e período escolar (matutino e noturno), com o intuito de estabelecer similaridades e diferença quanto as variáveis analisadas para os sexos e períodos.

\section{- Aspectos Éticos}

Considerando que este estudo envolveu diretamente seres humanos, foram tomados 
todos os cuidados éticos apropriados ao tipo de população investigada, conforme prevê as orientações éticas para pesquisas com seres humanos (resolução no 196 de 1996 do Conselho Nacional de Saúde do Ministério da Saúde) (BRASIL, 1996).

\section{RESULTADOS E DISCUSSÕES}

Do total de 371 de estudantes pesquisados, $71 \%(n=265)$ estavam matriculados no período matutino e $29 \%$ ( $n=106$ ) no turno noturno, sendo pouco mais da metade $(52,5 \%, n=195)$ do sexo feminino. Com relação ao turno matutino, conforme observado na tabela 1 , mais da metade dos alunos $(57 \%, n=151 \%)$ são do sexo feminino, sendo a maioria, solteiros $(94 \%, n=249)$, católicos (48\%, $n=128)$, não exercem atividade profissional $(63 \%, n=166)$ e possuem idade entre 14 a 34 anos. Quanto ao aspecto religiosidade, à maioria dos alunos do período matutino (43\%, $n=114$ ) se consideram religiosos e $36 \%(n=96)$ se dizem pouco religiosos (Tabela 1 ).

Tabela 1 - Perfil simplificado dos estudantes pesquisados, Colégio Estadual Professor Ivan Ferreira, Pires do Rio,

$$
\text { GO* }
$$

\begin{tabular}{|c|c|c|c|}
\hline \multirow{2}{*}{ Características } & \multicolumn{2}{|c|}{ Turnos } & \multirow{2}{*}{ Total } \\
\hline & Matutino $71 \%(n=265)$ & Noturno $29 \%(n=106)$ & \\
\hline \multicolumn{4}{|l|}{ Sexo } \\
\hline Masculino & $43 \%(n=114)$ & $58 \%(n=62)$ & $47,4 \%(n=176)$ \\
\hline Feminino & $57 \%(n=151)$ & $42 \%(n=44)$ & $52,5 \%(n=195)$ \\
\hline \multicolumn{4}{|l|}{ Estado civil } \\
\hline Solteiro & $94 \%(n=249)$ & $91 \%(n=96)$ & $92,9 \%(n=345)$ \\
\hline Casado & $4 \%(n=11)$ & $7 \%(n=8)$ & $5.1 \%(n=19)$ \\
\hline Divorciado/Separado & $1 \%(n=3)$ & $1 \%(n=1)$ & $1 \%(n=4)$ \\
\hline Não respondeu & $1 \%(n=2)$ & $1 \%(n=1)$ & $0,8 \%(n=3)$ \\
\hline \multicolumn{4}{|l|}{ Religião } \\
\hline Católico & $48 \%(n=128)$ & $49 \%(n=52)$ & $48,5 \%(n=180)$ \\
\hline Evangélico & $33 \%(n=87)$ & $32 \%(n=34)$ & $32,6 \%(n=121)$ \\
\hline Espírita & $2 \%(n=4)$ & $2 \%(n=2)$ & $1,6 \%(n=6)$ \\
\hline Outros & $2 \%(n=6)$ & $1 \%(n=1)$ & $1,8 \%(n=7)$ \\
\hline Não tem religião & $11 \%(n=29)$ & $16 \%(n=17)$ & $12,3 \%(n=46)$ \\
\hline Não respondeu & $4 \%(n=11)$ & - & $2,9 \%(n=11)$ \\
\hline \multicolumn{4}{|l|}{ Faixa etária } \\
\hline 14 a 15 & $17 \%(n=63)$ & $3,2 \%(n=12)$ & $20,2 \%(n=75)$ \\
\hline 16 a 17 & $31,5 \%(n=117)$ & $9,1 \%(n=34)$ & $40,7 \%(n=151)$ \\
\hline 18 a 19 & $12,1 \%(n=45)$ & $7,8 \%(n=29)$ & $20 \%(n=74)$ \\
\hline$>20$ & $6 \%(n=22)$ & $4,8 \%(n=18)$ & $10,7 \%(n=40)$ \\
\hline Não respondeu & $4,8 \%(n=18)$ & $3,5 \%(n=13)$ & $8,3 \%(n=31)$ \\
\hline \multicolumn{4}{|l|}{ Trabalha } \\
\hline Sim & $36 \%(n=96)$ & $66 \%(n=70)$ & $44,7 \%(n=166)$ \\
\hline Não & $63 \%(n=166)$ & $25 \%(n=26)$ & $51,7 \%(n=192)$ \\
\hline Não respondeu & $1 \%(n=3)$ & $9 \%(n=10)$ & $3,5 \%(n=13)$ \\
\hline \multicolumn{4}{|l|}{ Religiosidade } \\
\hline Muito religiosos & $10 \%(n=26)$ & $7 \%(n=7)$ & $88,9 \%(n=33)$ \\
\hline Religioso & $43 \%(n=114)$ & $37 \%(n=39)$ & $41,2 \%(n=153)$ \\
\hline Pouco religiosos & $36 \%(n=96)$ & $47 \%(n=50)$ & $39,3 \%(n=146)$ \\
\hline Sem religião & $8 \%(n=22)$ & $9 \%(n=10)$ & $8,6 \%(n=32)$ \\
\hline Não respondeu & $3 \%(n=7)$ & - & $1,8 \%(n=7)$ \\
\hline
\end{tabular}

*Os dados numéricos apresentados na tabela indicam o percentual dos estudantes seguido do número absoluto, entre parênteses. 
No turno noturno, a maioria dos alunos é do sexo masculino $(58 \%, n=62)$, solteiros $(91 \%$, $n=96)$, católicos $(9 \%, n=52)$, exercem alguma atividade profissional $(66 \%, n=70)$ e tem entre 15 a 24 anos. Quanto à religiosidade, a maioria dos alunos $(47 \% n=50)$ se auto avaliou como pouco religiosos $(43 \%, n=114)$ e $37 \%(n=39)$ como religiosos (Tabela 1$)$.

Quanto ao uso de drogas dentro da família dos jovens entrevistados, verificou-se que 49,3\% ( $n=183$ ) dos alunos afirmaram que possuíam algum parente que consome ou que já consumiu alguma droga. Entre os familiares dos alunos, que já tiveram contato com droga; os primos representam o maior percentual $(59 \%, n=108)$, seguido dos tios $(37,1 \%, n=68)$, pais $(16,3 \%, n=30)$, irmãos $(16,3 \%, n=30)$ e avós $(3,2 \%, n=12)$. Quando perguntados quais os tipos de drogas mais consumidas por esses familiares, as mais mencionadas foram às drogas de uso ilícito: maconha (56 referências), cocaína (25) e crack (33). As drogas lícitas: cigarro (37) e álcool (38), somadas, mostraram taxas inferiores. Um total de $26,2 \%(n=48)$ não respondeu a questão ${ }^{1}$.

Em relação à proporção geral de estudantes investigados, observou que pouco mais da metade $(52 \%, n=193)$ já teve contato, alguma vez na vida, com algum tipo de droga (lícita ou ilícita). Quanto à proporção de estudantes que já fizeram uso de drogas (lícitas ou ilícitas) por turno, observou-se que houve prevalência de uso no período matutino $31,5 \%(n=117)$, em relação ao período noturno $(20,4 \%, n=76)$. Contrariamente, no estudo de Martins et al. (2008), com 591 estudantes do ensino médio, em uma cidade do interior de São Paulo, o período noturno foi associado a um maior consumo de drogas quando comparado ao período diurno. No estudo destes autores, a média do consumo de drogas no período noturno chegou a um número expressivo de $70,5 \%$ do total de alunos investigados. Os achados do presente estudo, também divergiram do trabalho de Sanceverino e Abreu (2003), em Palhoça, Santa Catarina, onde foi observado, em uma amostra de 889 estudantes, maior consumo de drogas entre os estudantes que frequentavam aulas no período noturno.

Em relação ao gênero do total de estudantes investigados, verificou-se que o sexo masculino $(51 \%, n=99)$ apresentou pequena prevalência de consumo em comparação ao sexo feminino $(49 \%, n=94)$. Estes dados são similares aos apresentados em estudos realizados em diversas regiões do país, os quais evidenciam consumo maior (estatisticamente) de alguma substância psicoativa entre estudantes do sexo masculino (SILVA et al., 2006; MARTINS et al., 2008; PEREIRA et al., 2008; STRAUCH et al., 2009; CAMPOS et al., 2010; PINHEIRO et al., 2011; CAMPOS, 2011; NETO et al., 2012). Porém, no presente estudo, ao analisar as proporções dos estudantes que já fizeram uso de drogas (lícitas ou ilícitas) por turno, é possível observar que no período matutino a proporção de estudantes do sexo feminino foi superior (57\%), o que diverge um pouco dos estudos mencionados anteriormente. Estes dados podem estar relacionados ao maior uso de uso de drogas medicamentosas como anfetamínicos e ansiolíticos, tradicionalmente maior em estudantes do sexo feminino.

Quando observadas as taxas para o consumo de álcool, Tavares et al. (2001), em Pelotas, Rio Grande do Sul; Pereira et al. (2008) no Espírito Santo, encontraram prevalência similar entre os sexos. Em contraposição, Silva et al. (2006), em São José do Rio Preto, São Paulo; Horta et al., (2007) em Pelotas, Rio Grande do Sul; Souza et al. (2005), em Cuiabá, Mato Grosso e Carlini-

\footnotetext{
${ }^{1}$ Acreditamos que este dado possa ser reflexo da não compreensão das categorias gerais sobre o uso de drogas, por parte dos estudantes que responderam ao questionário aplicado. Tal observação constitui um possível problema quando da utilização de questionários autoaplicáveis.
} 
Cotrim (2000) em São Paulo, São Paulo, indicaram taxas superiores de consumo de álcool para o sexo masculino. Com relação ao tabaco, Tavares et al. (2001) e Silva et al (2006) observaram equilíbrio de uso entre os sexos; diferentemente de Muza et al. (1997), em Ribeirão Preto, São Paulo e Pereira et al. (2008), que relataram maior prevalência de uso no sexo masculino.

Quando os discentes foram indagados sobre quais os tipos de drogas que já consumiram alguma vez na vida, as drogas lícitas aparecem como as substâncias mais consumidas. A prevalência de consumo de álcool foi de $95,8 \%(n=185)$ e de tabaco foi de $46,1 \%(n=89)$. Além disso, no período matutino, houve maior prevalência de uso de álcool e tabaco $(61,3 \%, n=168)$, quando comparado ao noturno $(38,7 \%, n=106)$. Em Pelotas, Rio Grande do Sul, um estudo transversal, com 2.410 estudantes do ensino médio, mostrou que as drogas de uso lícito são mais amplamente utilizadas pelos adolescentes (TAVARES et al., 2001). Outros estudos realizados no Brasil também evidenciam maior prevalência de consumo de drogas lícitas entre adolescentes escolares (MUZA et al., 1997; GODOI et al., 1991; ALMEIDA FILHO et al., 2007), sendo o álcool a substância psicoativa mais consumida entre adolescentes escolares (PRADO et al., 2006; SILVA et al., 2006; ALMEIDA FILHO et al, 2007).

É necessário considerar, que esses resultados são esperados, uma vez, que o tabaco e as bebidas alcoólicas, pelo seu caráter lícito, fazem parte do cotidiano de muitas famílias e, portanto fazem parte da convivência e integração social dos jovens (ALMEIDA FILHO et al., 2007). Não obstante, ainda, advém destacar, o fato de campanhas publicitárias veicularem o uso de drogas lícitas a status social, promovendo à sociabilidade e aceitabilidade em relação a elas, que muitas vezes, provocam mais mortes e agravos à saúde quando comparado às demais drogas.

No que concerne às drogas ilícitas, a prevalência de uso entre os adolescentes alcançaram menores valores percentuais. É importante considerar que, apesar dos resultados ter apontado para uma baixa prevalência de experimentação/uso de drogas ilícitas na população estudada, importa salientar que tais drogas trazem problemas de grande magnitude, não só para aqueles que consomem, mas, também, para as pessoas que os cercam e para a sociedade de um modo geral (ALMEIDA FILHO et al., 2007). Na progressão do uso, a qual abarca desde a experimentação até o aparecimento dos problemas relacionados a este, as substâncias consumidas/usadas acabam por adquirir um papel progressivamente mais importante na vida dos usuários. Suas atividades e seu círculo social vão ficando cada vez mais associados ao uso, culminando, portanto, em problemas de natureza familiar, social, jurídico, financeiro e físicos, entre outros causados pela droga. Sobre este aspecto, alguns estudos (mais antigos e recentes) têm explorado de forma mais específica tal problemática (BUCHER, 1992; COTRIM, 1999; MORAES, 2008; SOUZA et al., 2013) e apontam para a necessidade urgente da adoção de políticas públicas específicas voltadas para ações preventivas abrangentes em detrimento de uma abordagem meramente repressiva e proibicionista.

Entre as drogas de uso ilícito, a maconha $(3,5 \%, n=13)$, a cocaína $(4 \%, n=07)$ o crack $(0,5 \%$, $n=2)$ e os solventes $(0,2 \%, n=01)$, configuram, nesta sequência, as substâncias mais mencionadas. Tavares et al. (2001), em seu estudo, indicaram, que entre as drogas de uso ilícito a maconha ocupou o primeiro lugar seguido dos solventes e medicamentos. Souza e Martins (1995), em Cuiabá, Mato Grosso, observaram que entre as substâncias ilícitas mais frequentemente usadas na vida pelos estudantes foram os solventes. Silva et al. (2006), em São José do Rio Preto, São Paulo, mostrou que os solventes ocupam o primeiro lugar das substâncias de uso ilícito seguido da maconha (SILVA et al., 2006). Para Almeida Filho et al., (2007) a maior prevalência de experimentação/uso de maconha, dentre as drogas ilícitas, se deve ao fato desta droga possuir, 
menor custo comercial, tornado-a mais acessível aos jovens oriundos de famílias de níveis sociais mais baixo, como é observado em muitos estudantes que participaram deste estudo.

Na presente pesquisa, verificou-se maior prevalência de uso de drogas ilícitas no sexo masculino (82\%, $n=19)$, quando comparado ao sexo feminino. Este dado mostra-se similar aos resultados obtidos por Muza et al. (1997), Silva et al. (2006), Tavares et al. (2001), os quais evidenciara maior prevalência de consumo de maconha e cocaína no sexo masculino.

Entre aqueles que consomem ou já consumiram algum tipo de droga, verificou-se que entre as causas apontadas como estímulo para o consumo destas substâncias destacam-se a influência dos amigos (15 referências), curiosidade (8), ansiedade e estresse (4), prazer e diversão (5) e ambiente familiar (3) o que corrobora com estudo correlato de Prado et al., (2006). No estudo de Carlini-Cotrim et al. (2000) com estudantes secundários de escolas estaduais e particulares da cidade de São Paulo, o consumo de alguma droga se deu sobretudo com amigos, em ambiente doméstico, festas e viagens. É importante mencionar que, na maioria das vezes é na própria família que os jovens efetuam seu primeiro contato com as drogas consideradas lícitas, como o álcool e o tabaco (SANCEVERINO e ABREU, 2003), pois as crianças, geralmente, convivem com o uso destas drogas em ambientes sócio-familiares, em situações de festividade e confraternização (ALMEIDA FILHO et al., 2007).

Para avaliar o desempenho escolar dos discentes diante ao uso de drogas, o presente estudo investigou, se os alunos tiveram alguma reprovação, no ensino médio, e se os mesmos já tiveram contato com alguma droga. Como pode ser observado na tabela 2 , verifica-se associação nítida para o uso de droga e reprovação escolar, uma vez que a frequência de reprovações é quase duas vezes maior naqueles alunos que já tiveram contato com drogas (lícitas ou ilícitas). No presente estudo verificou-se que $32,6 \%(n=121)$ dos alunos que já tiveram contato com alguma droga apresentaram reprovações escolares, enquanto, o número de reprovação, nos indivíduos que nunca experimentaram qualquer tipo de droga decresce para 17,2\% ( $n=64)$. Estes resultados confirmam que o consumo de drogas tem forte relação com o histórico de reprovações escolares.

Tabela 2 - Relação do uso de drogas com o número de reprovações, nos períodos estudados.

\begin{tabular}{ccc|cc}
\hline \multirow{2}{*}{ Período } & \multicolumn{2}{c|}{ Usou Drogas } & \multicolumn{2}{c}{ Não Usou Drogas } \\
& Reprovou & Não Reprovou & Reprovou & Não Reprovou \\
\hline Matutino & $17,2 \%(n=64)$ & $14,2 \%(n=53)$ & $13,2 \%(n=49)$ & $26,6 \%(n=99)$ \\
Noturno & $15,3 \%(n=57)$ & $5,1 \%(n=19)$ & $4 \%(n=15)$ & $4 \%(n=15)$ \\
Total & $32,6 \%(n=121)$ & $19,4 \%(n=72)$ & $17,2 \%(n=64)$ & $30,7 \%(n=114)$ \\
\hline
\end{tabular}

* Os dados numéricos apresentados na tabela indicam o percentual dos estudantes seguido do número absoluto, entre parênteses.

A associação entre uso de drogas e reprovação escolar também é referida nos estudos de Souza e Martins (1995), Tavares et al., (2001), Strauch et al. (2009) e Campos et al. (2011). Em recente estudo sobre uso de álcool entre estudantes do ensino médio noturno, em Coari, Amazonas, Campos et al. (2011) encontraram relação marcante com a prevalência de consumo de álcool em estudantes com o atraso escolar.

Ao se investigar a opinião dos discentes sobre quais estratégias que a escola poderia adotar para intervir e diminuir o uso de drogas no ambiente escolar, 45\% ( $n=167)$ dos estudantes 
assinalaram que palestras e campanhas, demonstrando os efeitos maléficos da droga na saúde ajudariam a minimizar o problema, $13,2 \%(n=49)$ referiram não saber o que poderia ser feito, $6,1 \%(n=23)$ responderam que a escola deveria revistar os alunos evitando a entrada de drogas na instituição, $2,4 \%(n=9)$ declararam que nada pode ser feito, $0,8 \%(n=3)$ considerou que a escola deveria proibir os professores de fazerem uso de tabaco na escola, o que denota um exemplo negativo diante dos discentes. $15,9 \% \quad(n=59)$ dos estudantes investigados não responderam a pergunta.

\section{CONCLUSÃO}

De acordo com os resultados obtidos, pode-se concluir que a prevalência de uso/experimentação de drogas entre os adolescentes escolares é alta, com maior prevalência no período matutino e no sexo masculino, sendo as drogas lícitas mais amplamente consumidas entre os jovens. Os índices de reprovação apresentados nos períodos investigados estão associados ao consumo de drogas, o que mostra que o uso destas substâncias interfere no desempenho dos alunos.

Nesse sentido, considerando que o uso de drogas é um problema de grande transcendência social, é requerida a adoção e/ou implementação de políticas de controle e combate a este uso, no âmbito de múltiplos setores da sociedade, tais como segurança pública, apoio social, saúde, educação, entre outros. No presente estudo, conclui-se também que a escola onde o estudo foi desenvolvido, tida como espaço privilegiado de aprendizagem, deve contribuir de forma mais efetiva com práticas de repulsão ao uso das drogas (lícitas ou ilícitas). As ações ou práticas que podem contribuir para que a escola participe de forma mais influente na superação do uso de drogas na adolescência são múltiplas. Dentre os aspectos que merecem destaque, citam-se: valorização da profissão docente e sua importância ou influência na instigação de reflexão sobre o uso indevido de drogas nas escolas e sua prevenção; capacitação dos docentes em relação aos métodos e técnicas de abordagem da problemática em sua prática cotidiana e estímulo à prática do educador aberto à realidade social da escola e sensível às necessidades dos estudantes. Tão importante também é eliminar ou reduzir obstáculos que incluem a conotação moral das drogas, colocadas no lugar de bode expiatório da sociedade atual, associada a baixas condições de trabalho de nossos docentes.

\section{REFERÊNCIAS BIBLIOGRÁFICAS}

1. Almeida Filho, A. J.; FERREIRA, M. A.; GOMES, M. L. B.; SILVA, R. C.; SANTOS, T. C. F. O adolescente e as drogas: consequências para a saúde. escente e drogas: Revista Enfermagem, v.11, n.4, p.605-10, 2007.

2. BRASIL. Ministério da Saúde, Conselho Nacional de Saúde. Resolução 196 de 10 de outubro de 1996. Diretrizes e normas regulamentadoras de pesquisa envolvendo seres humanos. Brasília. 1996.

3. BUCHER, R. Drogas e drogadição no Brasil. Porto Alegre: Artes Médicas, 1992.

4. CAMPOS, D. A., LIMA, H. S., CORRADI-WEBSTER, C. M., MORETTI-PIRES, R. O. O uso de álcool entre alunos do Ensino Médio noturno em um contexto amazônico. Revista Espaço para a Saúde, Londrina, v.13, n.1, p.15-22, 2011. 
5. CAMPOS, J. A. D. B.; ALMEIDA, J. C.; GARCIA, P. P. N.; FARIA, J. B. Consumo de álcool entre estudantes do ensino médio do município de Passos - MG. Ciência \& Saúde Coletiva, v.16, n.12, p.4745-4754, 2010.

6. CARLINI-COTRIM B., GAZAL-CARVALHO C., GOUVEIA N. Comportamento de saúde entre jovens estudantes das redes pública e privada da área metropolitana de Estado de São Paulo. Revista Saúde Publica, v.34, n.6, p.636-45, 2000.

7. COTRIM, B. C. A prevenção ao uso indevido de drogas nas escolas. In: Sudbrack MFO, organizadora. Prevenção ao uso indevido de drogas: diga sim à vida. v. 1. Brasília: CEAD/Unb; SENAD; 1999. p. 58-67.

8. GODOI, A. M.; MUZA, G. M.; COSTA, M. P.; GAMA, M. L. T. Consumo de substâncias psicoativas por escolares da rede privada do Distrito Federal. Rev. Saúde Pública , 25: 150-6, 1991.

9. HORTA, R. H., HORTA, B. L., PINHEIRO R. T., MORALES, B., STREY, M. N. Tabaco, álcool e outras drogas entre adolescentes em Pelotas, Rio Grande do Sul, Brasil: uma perspectiva de gênero. Caderno de Saúde Publica, v.23, n.4, p.775-83, 2007.

10. MALTA, D. C.; MASCARENHAS, M. D. M.; PORTO, D. L.; DUARTE, E. A.; SARDINHA, L. M.; BARRETO, S. M.; MORAIS NETO, O. L. Prevalência do consumo de álcool e drogas entre adolescentes: análise dos dados da Pesquisa Nacional de Saúde Escolar. Revista Brasileira de Epidemiologia, v.14, n.1, p. 136-46, 2011.

11. MARTINS, R. A., CRUZ, L. A. N., TEIXEIRA, P. S., MANZATO, A. J. Padrão de consumo de álcool entre estudantes do ensino médio de uma cidade do interior do estado de São Paulo. Revista Eletrônica Saúde Mental Álcool e Drogas. v.4, n.1, p 00-00, 2008.

12. MORAES, M. Integral healthcare model for treating problems caused by alcohol and other drugs: perceptions of users, their companions and practitioners. Ciência \& Saúde Coletiva, v. 13, n. 1, p. 121-133, 2008.

13. MUZA, G., BETTIOL, H., MUCHILLO, G., BARBIERI, M. A. Consumo de substâncias psicoativas por adolescentes escolares de Ribeirão Preto, SP (Brasil). I - Prevalência do consumo por sexo, idade e tipo de substância. Rev. Saúde Pública vol. 31 n. 1 São Paulo Feb. 1997

14. NETO, C., FRAGA, S., RAMOS, E. Consumo de substâncias lícitas por adolescentes portugueses. Revista Saúde Pública, v.46, n.5, p.808-15, 2012.

15. OBID - Observatório Brasileiro de Informações sobre Drogas. Informações sobre Drogas/ Definição e histórico. Ministério da Justiça do Brasil, 2007. Disponível em: http://www.obid.senad.gov.br. Acesso: 02 de abril de 2013.

16. PEREIRA, D. S.; SOUZA, R. S.; BUAIZ, V.; SIQUEIRA, M. M. Uso de substâncias psicoativas entre universitários de medicina da Universidade Federal do Espírito Santo. J Bras Psiquiatr. V. 57, n.3, p.188-195, 2008.

17. PINHEIRO, A., PICANÇO, P., BARBEITO, J. A realidade do consumo de drogas nas populações escolares. Rev. Portuguesa de Clínica Geral, v.27, n.4, 2011.

18. PRADO, D. S.; AZEREDO, F. S.; OLIVEIRA, T. B.; GARROTE, C. F. D. Uso de álcool, tabaco e drogas ilícitas por estudantes de farmácia da Universidade Federal de Goiás. Infarma , v.18, n. 11/12, 2006.

19. SAMPAIO, J. J. C., HERMETO, E. M. C., CARNEIRO, C. Atividades socioculturais e abandono do uso de drogas ilícitas: Percepção de adolescentes usuários em estudo de caso. Revista Baiana de Saúde Publica v.33, n.3, p.453-465, 2009. 
20. SANCEVERINO, S. L., ABREU, J. L. Aspectos epidemiológicos do uso de drogas entre estudantes do ensino médio no município de palhoça 2003. Ciência Saúde Coletiva v.9, n.4, p.1047-56, 2004.

21. SILVA, E. F., PAVANI, R. A. B., MORAES. M. S., CHIARAVALLOTI NETO, F. Prevalência do uso de drogas entre escolares do ensino médio do Município de São José do Rio Preto, São Paulo, Brasil. Caderno de Saúde Publica, v.22, n.6, p. 1151-1158, 2006.

22. SOUZA, D., P., O., MARTINS, D. T. O perfil epidemiológico do uso de drogas entre estudantes de 12 graus da rede estadual de ensino de Cuiabá, Brasil. Cadernos Saúde Pública, v.14, n.2, p.391-400, 1995.

23. SOUZA, D., P., O.; ARECO, K. N.; SILVEIRA FILHO, D. X. Álcool e alcoolismo entre adolescentes da rede estadual de ensino de Cuiabá, Mato Grosso. Rev Saude Publica. v.39, n.4, p.585-92, 2005.

24. SOUZA, M. M.; CARVALHO, R. N.; SILVA, L. R.; SANTOS, J. R.; AZEVEDO, E. B.; CAVALCANTI, P. B. Política nacional sobre drogas e saúde mental: percepções dos gestores e os desafios intersetoriais no arranjo político. Cadernos Brasileiros de Saúde Mental, v. 5, n. 11, p. 67-87, 2013.

25. STRAUCH, E. S.; PINHEIRO, R. T.; SILVA, R. A.; HORTA, B. L. Uso do álcool por adolescentes: estudo de base populacional. Revista de Saúde Pública, v.43, n.4, 2009.

26. STRAUCH, E. S.; PINHEIRO, R. T.; SILVA, R. A.; HORTA, B. L. Uso de álcool por adolescentes: estudo de base populacional. Revista Saúde Pública, v.43, n.4, p.647-55, 2009.

27. TAVARES, B. F.; BÉRIA, J. U.; LIMA, M. S. Fatores associados ao uso de drogas entre adolescentes escolares. Revista Saúde Pública, v.38, n.6, p.787-96, 2004.

28. TAVARES, B. F.; BÉRIA, J. U.; LIMA, M. S. Prevalência do uso de drogas e desempenho escolar entre adolescentes. Revista Saúde Pública, vol. 35 n.2, 2001 